\title{
MMW-NOTFALLCHECKLISTE
}

\section{Akutes Skrotum}

VON L. RINNAB
Abb. 1 Hämorrhagisch infarzierter Hoden nach kompletter Samenstrangtorsion (intraoperativer Befund).

\author{
Ein 19-jähriger Mann stellt sich wegen starken Schmerzen im Bereich \\ des rechten Hodens mit Projektion in die rechte Leiste nachmittags \\ in der urologischen Ambulanz vor. Die Symptomatik besteht seit \\ etwa zehn Stunden. Der Patient berichtet, dass er durch die heftigen \\ Schmerzen aufgewacht ist. Die Mictio ist subjektiv unauffällig.
}

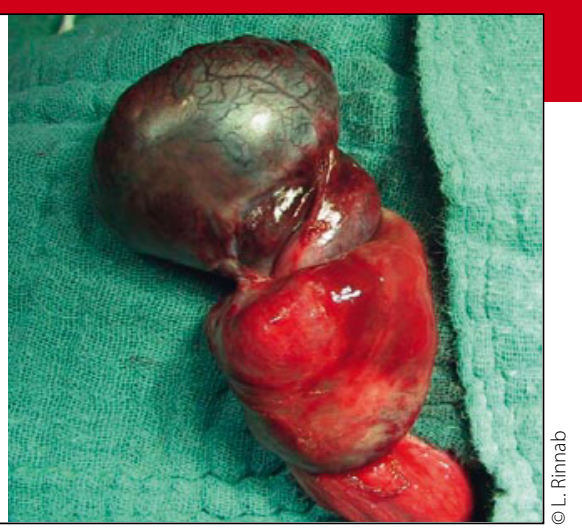

\section{Sofortdiagnostik}

- Die Inspektion zeigt einen geröteten, hochstehenden, stark druckdolenten Hoden sowie einen druckdolenten Samenstrang. Zudem besteht Übelkeit.

- Urinsticks und -sediment sind unauffällig (keine Leukozyt- und Bakteriurie). - Körpertemperatur rektal $37,2^{\circ} \mathrm{C}$.

- Sonografie: Der rechte Hoden zeigt ein inhomogenes Parenchym mit diskreter reaktiver Begleithydrozele. In der farbkodierten Duplexsonografie lassen sich keine Dopplersignale ableiten. Linker Hoden sonomorphologisch unauffällig.

\section{Differenzialdiagnostisch denken an:}

Hodentorsion, Hydatidentorsion (meist im Kindesalter), Epididymitis (häufig mit dysurischen Beschwerden, Fieber und Schüttelfrost können auftreten), Orchitis, inkarzerierte Hernie, Hodenruptur nach entsprechendem Hodentrauma, Hodentumor, unklare Bauchschmerzen (z.B. Appendizitis), infizierte Hydrozele, Spermatozele, extratestikuläre Störung (distaler Harnleiterstein). Selten: Mekoniumileus bei Neugeborenen, Fettgewebsnekrosen bei Pankreatitis, Wespenstich.

\section{Untersuchungen}

- Inspektion: hochstehender, geröteter, ödematös geschwollener Hoden. Gersches Zeichen: Einziehung der Skrotalhaut. Bei abdomineller Hodentorsion: leeres Skrotalfach, ggf. Abdominaltumor, akutes Abdomen.

- Palpation: Prehn'sches Zeichen: beim Anheben des Hodens kommt es zur
Schmerzverstärkung (DD: Epididymitis), der Hoden ist stark druckdolent, Hoden und Nebenhoden sind palpatorisch nicht mehr diskriminierbar.

- Urindiagnostik: Urinsticks, Urinsediment, ggf. Urinkultur.

- Paraklinische Untersuchung: Blutbild (insbesondere Leukozytenbestimmung, CRP, Blutgerinnung). B-Bild-Sonografie des Hodens. Power-Doppler-Sonografie und farbkodierte Duplexsonografie der A. und V. testicularis (immer im Vergleich zur Gegenseite).

\section{Therapeutische Maßnahmen}

- Klinisch besteht der V. a. eine Hodentorsion rechts, die innerhalb von vier bis sechs Stunden operiert werden muss (Hodenfreilegung). Sonst ist eine irreversible Schädigung des Keimepithels zu erwarten. Abhängig vom intraoperativen Befund ist einzeitig die kontralaterale Orchidopexie indiziert, da meist auch auf der kontralateralen Seite durch

\section{Kasuistik - so ging es weiter}

Bei klinischem V. a. eine Hodentorsion rechts erfolgte eine Hodenfreilegung rechts. Intraoperativ zeigte sich eine Hodentorsion mit hämorrhagischer Infarzierung (s. Abb. 1). Trotz sofortiger Detorquierung kam es nicht zur sichtbaren Revaskularisation des Hodens, sodass eine Ablatio testis und zusätzlich eine kontralaterale Orchidopexie erfolgte. Der Patient wurde am Folgetag bei reizlosen Wundverhältnissen entlassen. eine abnorme Mobilität des Hodens die anatomische Gegebenheit für eine Samenstrangstorsion vorliegt.

- Bei klinisch eindeutiger Epididymitis erfolgt eine antibiotische und symptomatische Therapie, z. B. mit dem Gyrasehemmer Tavanic ${ }^{\oplus}$ per os und Hochlagerung mit lokaler Kühlung.

- Bei zweifelsfreier Hydatidentorsion („blue dot sign“) kann ggf. auf die Hodenfreilegung verzichtet und eine konservative Behandlung mit Antiphlogistika (NSAR) durchgeführt werden. - Bei V. a. einen eingebluteten Hodentumor sollte die Hodenfreilegung über einen inguinalen Zugangsweg erfolgen. Zuvor ist die Bestimmung der Hodentumormarker obligat! Da i. d. R. kein akuter Handlungsbedarf besteht, können die Fragen nach Kryokonservierung des Ejakulates geklärt werden.

- Liegt ein Hodentrauma mit fraglich oder nachgewiesener Tunica-albugineaRuptur vor, sollte der Hoden unverzüglich freigelegt werden.

\section{Fehlerquellen}

Verzögerung der Diagnostik und längere analgetische und antibiotische Behandlung. Patient mit akutem Skrotum rasch bei einem Urologen oder in einer urologischen Klinik vorstellen.

\section{Anschrift des Verfassers:}

Priv.-Doz. Dr. med. habil. Ludwig Rinnab Urologische Praxis Neu-Ulm, Praxisschwerpunkt Uro-Onkologie/Andrologie Augsburgerstraße 1 a, D-89231 Neu-Ulm, E-Mail: ludwig@dr-rinnab.de 